\title{
Terminkalender 2021
}

https://doi.org/10.1515/iwp-2020-2143

\begin{tabular}{|c|c|c|}
\hline $\begin{array}{l}\text { 11. bis } 13 . \text { Januar } \\
\text { online }\end{array}$ & $\begin{array}{l}\text { APE } 2021 \text { Academic Publishing in Europe } \\
\text { The New Face of Trust }\end{array}$ & $\begin{array}{l}\text { Berlin Institute for Scholarly Publishing (BISP) gGmbH, Lützowstraße 33, } \\
10785 \text { Berlin, www.berlinstitute.org, www.ape2021.eu }\end{array}$ \\
\hline $\begin{array}{l}\text { 19. bis } 20 \text { Januar } \\
\text { online }\end{array}$ & $\begin{array}{l}\text { DGI-Onlineseminar } \\
\text { Kommunikation für Information Professionals } \\
\text { Modul I }\end{array}$ & $\begin{array}{l}\text { DGI-Geschäftsstelle, Windmühlstraße 3, } 60329 \text { Frankfurt am Main, } \\
\text { Telefon } 069 \text { 430313, Fax } 069 \text { 4909096, mail@dgi-info.de, } \\
\text { www.dgi-info.de }\end{array}$ \\
\hline $\begin{array}{l}\text { 21. Januar } \\
\text { online }\end{array}$ & $\begin{array}{l}\text { DGI-Onlineseminar } \\
\text { Kommunikation für Information Professionals } \\
\text { Modul II }\end{array}$ & $\begin{array}{l}\text { DGI-Geschäftsstelle, Windmühlstraße 3, } 60329 \text { Frankfurt am Main, } \\
\text { Telefon } 069 \text { 430313, Fax } 069 \text { 4909096, mail@dgi-info.de, } \\
\text { www.dgi-info.de }\end{array}$ \\
\hline $\begin{array}{l}\text { 21. bis 22. Januar } \\
\text { online }\end{array}$ & $\begin{array}{l}\text { BOBCATSSS } 2021 \\
\text { Digital Transformation }\end{array}$ & https://bobcatsss2021.sciencesconf.org/ \\
\hline $\begin{array}{l}\text { 26. bis } 27 . \text { Januar } \\
\text { online }\end{array}$ & $\begin{array}{l}\text { DGI-Onlineseminar } \\
\text { Projektplanung, -durchführung und } \\
\text {-evaluierung } \\
\text { Modul I }\end{array}$ & $\begin{array}{l}\text { DGI-Geschäftsstelle, Windmühlstraße 3, } 60329 \text { Frankfurt am Main, } \\
\text { Telefon } 069 \text { 430313, Fax } 069 \text { 4909096, mail@dgi-info.de, } \\
\text { www.dgi-info.de }\end{array}$ \\
\hline $\begin{array}{l}\text { 28. Januar } \\
\text { online }\end{array}$ & $\begin{array}{l}\text { DGI-Onlineseminar } \\
\text { Projektplanung, -durchführung und } \\
\text {-evaluierung } \\
\text { Modul II }\end{array}$ & $\begin{array}{l}\text { DGI-Geschäftsstelle, Windmühlstraße 3, } 60329 \text { Frankfurt am Main, } \\
\text { Telefon } 069 \text { 430313, Fax } 069 \text { 4909096, mail@dgi-info.de, } \\
\text { www.dgi-info.de }\end{array}$ \\
\hline $\begin{array}{l}\text { 29. Januar } \\
\text { online }\end{array}$ & $\begin{array}{l}\text { DGI-Onlineseminar } \\
\text { Schreibwerkstatt I - Websites }\end{array}$ & $\begin{array}{l}\text { DGI-Geschäftsstelle, Windmühlstraße 3, } 60329 \text { Frankfurt am Main, } \\
\text { Telefon } 069 \text { 430313, Fax } 069 \text { 4909096, mail@dgi-info.de, } \\
\text { www.dgi-info.de }\end{array}$ \\
\hline $\begin{array}{l}\text { 1. Februar } 2021 \\
\text { online }\end{array}$ & $\begin{array}{l}\text { DGI-Onlineseminar } \\
\text { Schreibwerkstatt II - Blogs }\end{array}$ & $\begin{array}{l}\text { DGI-Geschäftsstelle, Windmühlstraße 3, } 60329 \text { Frankfurt am Main, } \\
\text { Telefon } 069 \text { 430313, Fax } 069 \text { 4909096, mail@dgi-info.de, } \\
\text { www.dgi-info.de }\end{array}$ \\
\hline $\begin{array}{l}\text { 2. bis 3. Februar } \\
\text { online }\end{array}$ & $\begin{array}{l}\text { DGI-Onlineseminar } \\
\text { Formale und Inhaltliche } \\
\text { Informationserschließung } \\
\text { Modul I }\end{array}$ & $\begin{array}{l}\text { DGI-Geschäftsstelle, Windmühlstraße 3, } 60329 \text { Frankfurt am Main, } \\
\text { Telefon } 069 \text { 430313, Fax } 069 \text { 4909096, mail@dgi-info.de, } \\
\text { www.dgi-info.de }\end{array}$ \\
\hline $\begin{array}{l}\text { 4. Februar } \\
\text { online }\end{array}$ & $\begin{array}{l}\text { DGI-Onlineseminar } \\
\text { Formale und Inhaltliche } \\
\text { Informationserschließung } \\
\text { Modul II }\end{array}$ & $\begin{array}{l}\text { DGI-Geschäftsstelle, Windmühlstraße 3, } 60329 \text { Frankfurt am Main, } \\
\text { Telefon } 069 \text { 430313, Fax } 069 \text { 4909096, mail@dgi-info.de, } \\
\text { www.dgi-info.de }\end{array}$ \\
\hline $\begin{array}{l}\text { 2. bis } 4 \text {. Februar } \\
\text { Karlsruhe }\end{array}$ & $\begin{array}{l}\text { LEARNTEC } 2021 \\
\text { 29. Internationale Fachmesse und Kongress }\end{array}$ & $\begin{array}{l}\text { Karlsruher Messe- und Kongress-GmbH, Festplatz9, } 76137 \text { Karlsruhe, } \\
\text { Telefon 07213720-0, Fax 07213720-2116, info@kmkg.de, } \\
\text { www.learntec.de }\end{array}$ \\
\hline $\begin{array}{l}\text { 8. Februar } \\
\text { online }\end{array}$ & $\begin{array}{l}\text { DGI-Onlineseminar } \\
\text { Schreibwerkstatt III - Storytelling }\end{array}$ & $\begin{array}{l}\text { DGI-Geschäftsstelle, Windmühlstraße 3, } 60329 \text { Frankfurt am Main, } \\
\text { Telefon } 069 \text { 430313, Fax } 069 \text { 4909096, mail@dgi-info.de, } \\
\text { www.dgi-info.de }\end{array}$ \\
\hline $\begin{array}{l}\text { 9. Februar } \\
\text { online }\end{array}$ & $\begin{array}{l}\text { DGI-Onlineseminar } \\
\text { Schreibwerkstatt IV - Essays }\end{array}$ & $\begin{array}{l}\text { DGI-Geschäftsstelle, Windmühlstraße 3, } 60329 \text { Frankfurt am Main, } \\
\text { Telefon } 069 \text { 430313, Fax } 069 \text { 4909096, mail@dgi-info.de, } \\
\text { www.dgi-info.de }\end{array}$ \\
\hline $\begin{array}{l}\text { 15. Februar } \\
\text { online }\end{array}$ & $\begin{array}{l}\text { DGI-Onlineseminar } \\
\text { Digitalfotografie für Social Media und } \\
\text { Websites I }\end{array}$ & $\begin{array}{l}\text { DGI-Geschäftsstelle, Windmühlstraße 3, } 60329 \text { Frankfurt am Main, } \\
\text { Telefon } 069 \text { 430313, Fax } 069 \text { 4909096, mail@dgi-info.de, } \\
\text { www.dgi-info.de }\end{array}$ \\
\hline $\begin{array}{l}\text { 16. Februar } \\
\text { online }\end{array}$ & $\begin{array}{l}\text { DGI-Onlineseminar } \\
\text { Digitalfotografie für Social Media und } \\
\text { Websites II }\end{array}$ & $\begin{array}{l}\text { DGI-Geschäftsstelle, Windmühlstraße 3, } 60329 \text { Frankfurt am Main, } \\
\text { Telefon } 069 \text { 430313, Fax } 069 \text { 4909096, mail@dgi-info.de, } \\
\text { www.dgi-info.de }\end{array}$ \\
\hline
\end{tabular}




\begin{tabular}{|c|c|c|}
\hline $\begin{array}{l}\text { 17. Februar } \\
\text { online }\end{array}$ & $\begin{array}{l}\text { DGI-Onlineseminar } \\
\text { Social Media und Öffentlichkeitsarbeit I - } \\
\text { Einführung }\end{array}$ & $\begin{array}{l}\text { DGI-Geschäftsstelle, Windmühlstraße 3, } 60329 \text { Frankfurt am Main, } \\
\text { Telefon } 069 \text { 430313, Fax } 069 \text { 4909096, mail@dgi-info.de, } \\
\text { www.dgi-info.de }\end{array}$ \\
\hline $\begin{array}{l}\text { 18. Februar } \\
\text { online }\end{array}$ & $\begin{array}{l}\text { DGI-Onlineseminar } \\
\text { Social Media und Öffentlichkeitsarbeit II - } \\
\text { Vertiefung }\end{array}$ & $\begin{array}{l}\text { DGI-Geschäftsstelle, Windmühlstraße 3, } 60329 \text { Frankfurt am Main, } \\
\text { Telefon } 069 \text { 430313, Fax } 069 \text { 4909096, mail@dgi-info.de, } \\
\text { www.dgi-info.de }\end{array}$ \\
\hline $\begin{array}{l}\text { 17. bis } 18 . \text { Februar } \\
\text { online }\end{array}$ & Open Science Conference 2021 & $\begin{array}{l}\text { Dr. Doreen Siegfried, ZBW-Leibniz-Informationszentrum Wirtschaft, } \\
\text { Düsternbrooker Weg 120, } 24105 \text { Kiel, osc@zbw.eu, osc@zbw.eu, } \\
\text { www.open-science-conference.eu/ }\end{array}$ \\
\hline $\begin{array}{l}\text { 23. bis } 24 \text {. Februar } \\
\text { online }\end{array}$ & $\begin{array}{l}\text { DGI-Onlineseminar } \\
\text { Projektplanung, -durchführung und } \\
\text {-evaluierung } \\
\text { Modul I }\end{array}$ & $\begin{array}{l}\text { DGI-Geschäftsstelle, Windmühlstraße 3, } 60329 \text { Frankfurt am Main, } \\
\text { Telefon } 069 \text { 430313, Fax } 069 \text { 4909096, mail@dgi-info.de, } \\
\text { www.dgi-info.de }\end{array}$ \\
\hline $\begin{array}{l}\text { 25. Februar } \\
\text { online }\end{array}$ & $\begin{array}{l}\text { DGI-Onlineseminar } \\
\text { Projektplanung, -durchführung und } \\
\text {-evaluierung } \\
\text { Modul II }\end{array}$ & $\begin{array}{l}\text { DGI-Geschäftsstelle, Windmühlstraße 3, } 60329 \text { Frankfurt am Main, } \\
\text { Telefon } 069 \text { 430313, Fax } 069 \text { 4909096, mail@dgi-info.de, } \\
\text { www.dgi-info.de }\end{array}$ \\
\hline $\begin{array}{l}\text { 25. Februar } \\
\text { online }\end{array}$ & Global DIGITAL FUTUREcongress & www.digital-futurecongress.de \\
\hline $\begin{array}{l}\text { 25. Februar } \\
\text { online }\end{array}$ & DVMD-Fokus: Aus- und Weiterbildung & $\begin{array}{l}\text { Der Fachverband für Dokumentation und Informationsmanagement } \\
\text { in der Medizin e.V., Lobdengaustraße 1, } 69493 \text { Hirschberg, } \\
\text { dvmd@dvmd.de }\end{array}$ \\
\hline $\begin{array}{l}\text { 2. bis 3. März } \\
\text { online }\end{array}$ & $\begin{array}{l}\text { DGI-Onlineseminar } \\
\text { Formale und Inhaltliche } \\
\text { Informationserschließung } \\
\text { Modul I }\end{array}$ & $\begin{array}{l}\text { DGI-Geschäftsstelle, Windmühlstraße 3, } 60329 \text { Frankfurt am Main, } \\
\text { Telefon } 069 \text { 430313, Fax } 069 \text { 4909096, mail@dgi-info.de, } \\
\text { www.dgi-info.de }\end{array}$ \\
\hline $\begin{array}{l}\text { 4. März } \\
\text { online }\end{array}$ & $\begin{array}{l}\text { DGI-Onlineseminar } \\
\text { Formale und Inhaltliche } \\
\text { Informationserschließung } \\
\text { Modul II }\end{array}$ & $\begin{array}{l}\text { DGI-Geschäftsstelle, Windmühlstraße 3, } 60329 \text { Frankfurt am Main, } \\
\text { Telefon } 069 \text { 430313, Fax } 069 \text { 4909096, mail@dgi-info.de, } \\
\text { www.dgi-info.de }\end{array}$ \\
\hline $\begin{array}{l}\text { 8. bis } 10 . \text { März } \\
\text { Regensburg }\end{array}$ & $\begin{array}{l}\text { 16. Internationales Symposium für } \\
\text { Informationswissenschaft } \\
\text { Information zwischen Daten und Wissen: Die } \\
\text { Informationswissenschaft und ihre Nachbarn } \\
\text { von Data Science bis Digital Humanities }\end{array}$ & $\begin{array}{l}\text { Prof. Dr. Christian Wolff, Universität Regensburg, Institut für Medien-, } \\
\text { Informations- und Kulturwissenschaft (IMIK), Universitätsstraße 31, } \\
93053 \text { Regensburg, christian.wolff@sprachlit.uni-regensburg.de, } \\
\text { www.isi2021.de }\end{array}$ \\
\hline $\begin{array}{l}\text { 11. März } \\
\text { online }\end{array}$ & DVMD-Fokus: Datenmanagement & $\begin{array}{l}\text { Der Fachverband für Dokumentation und Informationsmanagement in } \\
\text { der Medizin e.V., Lobdengaustraße 1, } 69493 \text { Hirschberg, } \\
\text { dvmd@dvmd.de }\end{array}$ \\
\hline $\begin{array}{l}\text { 16. März } \\
\text { online }\end{array}$ & $\begin{array}{l}\text { DGI-Onlineseminar } \\
\text { Wissensmanagement - Einführung für } \\
\text { Information Professionals } \\
\text { Modul I }\end{array}$ & $\begin{array}{l}\text { DGI-Geschäftsstelle, Windmühlstraße 3, } 60329 \text { Frankfurt am Main, } \\
\text { Telefon } 069 \text { 430313, Fax } 069 \text { 4909096, mail@dgi-info.de, } \\
\text { www.dgi-info.de }\end{array}$ \\
\hline $\begin{array}{l}\text { 17. März } \\
\text { online }\end{array}$ & $\begin{array}{l}\text { DGI-Onlineseminar } \\
\text { Wissensmanagement - Wissensmanagement } \\
\text { in der Praxis } \\
\text { Modul II }\end{array}$ & $\begin{array}{l}\text { DGI-Geschäftsstelle, Windmühlstraße 3, } 60329 \text { Frankfurt am Main, } \\
\text { Telefon } 069 \text { 430313, Fax } 069 \text { 4909096, mail@dgi-info.de, } \\
\text { www.dgi-info.de }\end{array}$ \\
\hline $\begin{array}{l}\text { 18. März } \\
\text { online }\end{array}$ & $\begin{array}{l}\text { DGI-Onlineseminar } \\
\text { Social Media und Öffentlichkeitsarbeit I- } \\
\text { Einführung }\end{array}$ & $\begin{array}{l}\text { DGI-Geschäftsstelle, Windmühlstraße 3, } 60329 \text { Frankfurt am Main, } \\
\text { Telefon } 069 \text { 430313, Fax } 069 \text { 4909096, mail@dgi-info.de, } \\
\text { www.dgi-info.de }\end{array}$ \\
\hline $\begin{array}{l}\text { 19. März } \\
\text { online }\end{array}$ & $\begin{array}{l}\text { DGI-Onlineseminar } \\
\text { Social Media und Öffentlichkeitsarbeit II - } \\
\text { Vertiefung }\end{array}$ & $\begin{array}{l}\text { DGI-Geschäftsstelle, Windmühlstraße 3, } 60329 \text { Frankfurt am Main, } \\
\text { Telefon } 069 \text { 430313, Fax } 069 \text { 4909096, mail@dgi-info.de, } \\
\text { www.dgi-info.de }\end{array}$ \\
\hline $\begin{array}{l}\text { 17. bis 31. März } \\
\text { online }\end{array}$ & $\begin{array}{l}\text { iConference } \\
\text { Diversity, Divergence, Dialogue }\end{array}$ & https://ischools.org/iConference \\
\hline
\end{tabular}




\begin{tabular}{|c|c|c|}
\hline $\begin{array}{l}\text { 22. bis } 26 . \text { März } \\
\text { online }\end{array}$ & DIGITAL FUTUREcongress & www.digital-futurecongress.de \\
\hline $\begin{array}{l}\text { 23. bis } 27 . \text { März } \\
\text { Stuttgart }\end{array}$ & didacta 2021 & $\begin{array}{l}\text { Landesmesse Stuttgart GmbH, Messepiazza 1, } 70629 \text { Stuttgart, } \\
\text { Telefon } 04971118560 \text { 0, info@messe-stuttgart.de, } \\
\text { https://www.messe-stuttgart.de/didacta/ }\end{array}$ \\
\hline $\begin{array}{l}\text { 26. März } \\
\text { Berlin/online }\end{array}$ & 2. Bibliothekspolitischer Bundeskongress & $\begin{array}{l}\text { Deutscher Bibliotheksverband e.V. (dbv), Fritschestraße 27-28, } \\
10585 \text { Berlin, Telefon } 0306449899 \text { 10, Fax } 0306449899 \text { 29, } \\
\text { dbv@bibliotheksverband.de, http://www.bibliotheksverband.de/ }\end{array}$ \\
\hline $\begin{array}{l}\text { 14. April } \\
\text { online }\end{array}$ & $\begin{array}{l}\text { DGI-Onlineseminar } \\
\text { Social Media und Recherche - Einführung }\end{array}$ & $\begin{array}{l}\text { DGI-Geschäftsstelle, Windmühlstraße 3, } 60329 \text { Frankfurt am Main, } \\
\text { Telefon } 069 \text { 430313, Fax } 069 \text { 4909096, mail@dgi-info.de, } \\
\text { www.dgi-info.de }\end{array}$ \\
\hline $\begin{array}{l}\text { 2. bis 3. Mai } \\
\text { Brandenburg }\end{array}$ & DMB Jahrestagung & $\begin{array}{l}\text { Deutscher Museumsbund e. V., In der Halde 1, } 14195 \text { Berlin, Telefon } \\
030 \text { 841095-17, Fax } 030 \text { 841095-19, office@museumsbund.de, } \\
\text { www.museumsbund.de/aktuelles/jahrestagung/ }\end{array}$ \\
\hline $\begin{array}{l}\text { 3. bis 5. Mai } \\
\text { Mannheim }\end{array}$ & $\begin{array}{l}\text { 17. DTT-Symposion } 2021 \text { „Terminologie: } \\
\text { Industrie, Information, Intelligenz“ }\end{array}$ & $\begin{array}{l}\text { Deutscher Terminologie-Tag e.V. (DTT), Geschäftsstelle c/o Technische } \\
\text { Hochschule Köln, Institut für Informationsmanagement (IIM), } \\
\text { Claudiusstraße 1, } 50768 \text { Köln, geschaeftsstelle@dttev.org, http:// } \\
\text { dttev.org/aktuelles-terminologie-erleben/2020/223-dtt-symposion- } \\
\text { 2021.html }\end{array}$ \\
\hline $\begin{array}{l}\text { 5. Mai } \\
\text { Brandenburg }\end{array}$ & $\begin{array}{l}\text { Frühjahrstagung der Fachgruppe } \\
\text { Dokumentation }\end{array}$ & $\begin{array}{l}\text { Frank von Hagel, Institut für Museumsforschung, In der Halde 1, } \\
14195 \text { Berlin, Telefon: } 030 \text { 8301-460, Fax: } 030 \text { 8301-504, E-Mail: } \\
\text { f.v.hagel@smb.spk-berlin.de, www.museumsbund.de }\end{array}$ \\
\hline $\begin{array}{l}\text { 19. bis } 20 . \text { Mai } \\
\text { online }\end{array}$ & $\begin{array}{l}\text { B2B Media Days } 2021 \text { - Kongress der } \\
\text { Deutschen Fachpresse }\end{array}$ & $\begin{array}{l}\text { Yvonne Barnes, Verein Deutsche Fachpresse, Markgrafenstraße 15, } \\
10969 \text { Berlin, Telefon } 030726298 \text { 140, barnes@deutsche-fachpresse. } \\
\text { de www.b2b-media-days.de }\end{array}$ \\
\hline $\begin{array}{l}\text { 9. bis } 11 . \text { Juni } \\
\text { Ilmenau }\end{array}$ & $\begin{array}{l}\text { PATINFO } 2021 \\
\text { 43. Kolloquium der Technischen Universität } \\
\text { Ilmenau über Patentinformation }\end{array}$ & $\begin{array}{l}\text { Astrid Schieck, Technische Universität Ilmenau, PATON, } \\
\text { Landespatentzentrum Thüringen, Postfach } 1005 \text { 65, } 98684 \text { Ilmenau, } \\
\text { Telefon } 03677 \text { 69-4572, Fax 03677 69-4538, patinfo@tu-ilmenau.de, } \\
\text { www.paton.tu-ilmenau.de }\end{array}$ \\
\hline $\begin{array}{l}\text { 15. bis 18. Juni } \\
\text { Bremen }\end{array}$ & 109. Bibliothekartag & $\begin{array}{l}\text { K.I.T. Group GmbH, Kurfürstendamm 71, } 10709 \text { Berlin Deutschland, } \\
\text { Telefon } 030246 \text { 03-260, bibliothekartag2020@kit-group.org, }\end{array}$ \\
\hline $\begin{array}{l}\text { 23. bis } 25 \text {. Juni } \\
\text { online }\end{array}$ & 50. LIBER-Konferenz & $\begin{array}{l}\text { LIBER c/o Koninklijke Bibliotheek, (Nationalbibliothek der } \\
\text { Niederland), Postfach 90407, } 2509 \text { LK Den Haag, Niederlande, } \\
\text { Telefon +31 } 70314 \text { 0767, liber@kb.nl }\end{array}$ \\
\hline $\begin{array}{l}\text { 12. Juli } \\
\text { online }\end{array}$ & $\begin{array}{l}\text { DGI-Onlineseminar } \\
\text { Social Media und Recherche - Einführung }\end{array}$ & $\begin{array}{l}\text { DGI-Geschäftsstelle, Windmühlstraße 3, } 60329 \text { Frankfurt am Main, } \\
\text { Telefon } 069 \text { 430313, Fax } 069 \text { 4909096, mail@dgi-info.de, } \\
\text { www.dgi-info.de }\end{array}$ \\
\hline $\begin{array}{l}\text { 6. bis } 9 . \text { September } \\
\text { Amsterdam, } \\
\text { Niederlande }\end{array}$ & $\begin{array}{l}\text { SEMANTiCS } 2021 \text { - In the Era of Knowledge } \\
\text { Graphs }\end{array}$ & $\begin{array}{l}\text { Semantic Web Company, R\&I Department, Neubaugasse 1, } 1070 \text { Wien, } \\
\text { Österreich, https://2019.semantics.cc/conference }\end{array}$ \\
\hline $\begin{array}{l}\text { 27. September } \\
\text { online }\end{array}$ & $\begin{array}{l}\text { DGI-Onlineseminar } \\
\text { Social Media und Recherche - Einführung }\end{array}$ & $\begin{array}{l}\text { DGI-Geschäftsstelle, Windmühlstraße 3, } 60329 \text { Frankfurt am Main, } \\
\text { Telefon } 069 \text { 430313, Fax } 069 \text { 4909096, mail@dgi-info.de, } \\
\text { www.dgi-info.de }\end{array}$ \\
\hline $\begin{array}{l}\text { 11. bis } 13 . \text { Oktober } \\
\text { Berlin }\end{array}$ & $\begin{array}{l}\text { Berliner Herbsttreffen der } \\
\text { Museumsdokumentation }\end{array}$ & $\begin{array}{l}\text { Frank von Hagel, Institut für Museumsforschung, In der Halde 1, } \\
14195 \text { Berlin, Telefon: (030) 8301-460, Fax: (030) 8301-504, E-Mail: } \\
\text { f.v.hagel@smb.spk-berlin.de, www.museumsbund.de }\end{array}$ \\
\hline $\begin{array}{l}\text { 20. bis } 24 . \text { Oktober } \\
\text { Frankfurt am Main }\end{array}$ & $\begin{array}{l}\text { 73. Frankfurter Buchmesse } \\
\text { Ehrengast Spanien }\end{array}$ & $\begin{array}{l}\text { Ausstellungs- und Messe GmbH, Frankfurter Buchmesse, } \\
\text { Braubachstraße 16, 60311 Frankfurt am Main, Telefon } 069 \text { 2102-0, } \\
\text { Fax } 069 \text { 2102-227/-277, info@book-fair.com, www.buchmesse.de }\end{array}$ \\
\hline $\begin{array}{l}\text { 26. bis 28. Oktober } \\
\text { Bern, Schweiz }\end{array}$ & Schweizer Bibliothekskongress 2021 & $\begin{array}{l}\text { Bibliosuisse, Bleichemattstrasse 42, } 5000 \text { Aarau, Schweiz, } \\
\text { Telefon +41 } 62823 \text { 1938, Fax +41 } 62823 \text { 1939, info@bibliosuisse.ch, } \\
\text { info@bis.ch }\end{array}$ \\
\hline
\end{tabular}

